\title{
Gene dosage affects the cardiac and brain phenotype in nonmuscle myosin II-B-depleted mice
}

\author{
Deniz Üren, ${ }^{1}$ Hweung-Kon Hwang, ${ }^{1}$ Yoshinobu Hara, ${ }^{1}$ Kazuyo Takeda, ${ }^{2}$ \\ Sachiyo Kawamoto, ${ }^{1}$ Antonella N. Tullio, ${ }^{1} \mathrm{Zu}-\mathrm{Xi}$ Yu, ${ }^{2}$ Victor J. Ferrans, ${ }^{2}$ \\ Nancy Tresser, ${ }^{3}$ Alexander Grinberg, ${ }^{4}$ Yvette A. Preston, ${ }^{1}$ and Robert S. Adelstein ${ }^{1}$ \\ ${ }^{1}$ Laboratory of Molecular Cardiology, National Heart, Lung, and Blood Institute, \\ ${ }^{2}$ Pathology Section, National Heart, Lung, and Blood Institute, \\ ${ }^{3}$ Office of the Clinical Director, National Institute of Neurological Disorders and Stroke, and \\ ${ }^{4}$ Laboratory of Mammalian Genes and Development, National Institute of Child Health and Human Development, \\ National Institutes of Health, Bethesda, Maryland 20892, USA
}

Address correspondence to: Robert S. Adelstein, National Institutes of Health, Building 10, Room 8N202,

10 Center Drive, Bethesda, Maryland 20892-1762, USA.

Phone: (301) 496-1865; Fax: (301) 402-1542; E-mail: AdelsteR@NHLBI.NIH.GOV.

Deniz Üren, Hweung-Kon Hwang, Yoshinobu Hara, and Kazuyo Takeda each made major contributions to this work.

Received for publication August 18, 1999, and accepted in revised form January 25, 2000.

Complete ablation of nonmuscle myosin heavy chain II-B (NMHC-B) in mice resulted in cardiac and brain defects that were lethal during embryonic development or on the day of birth. In this paper, we report on the generation of mice with decreased amounts of NMHC-B. First, we generated $B^{\Delta I} / B^{\Delta I}$ mice by replacing a neural-specific alternative exon with the PGK-Neo cassette. This resulted in decreased amounts of NMHC-B in all tissues, including a decrease of $88 \%$ in the heart and $65 \%$ in the brain compared with $B^{+} / B^{+}$tissues. $B^{\Delta I} / B^{\Delta I}$ mice developed cardiac myocyte hypertrophy between 7 months and 11 months of age, at which time they reexpressed the cardiac $\beta$-MHC. Serial sections of $B^{\Delta I} / B^{\Delta I}$ brains showed abnormalities in neural cell migration and adhesion in the ventricular wall. Crossing $B^{\Delta I} / B^{\Delta I}$ with $B^{+} / B^{-}$mice generated $B^{\Delta I} / B^{-}$mice, which showed a further decrease of approximately $55 \%$ in NMHC-B in the heart and brain compared with $B^{\Delta I} / B^{\Delta I}$ mice. Five of $8 B^{\Delta I} / B^{-}$mice were born with a membranous ventricular septal defect. Moreover, 5 of $5 B^{\Delta I} / B^{-}$mice developed myocyte hypertrophy by 1 month; $B^{\Delta I} / B^{-}$mice also reexpressed the cardiac $\beta$-MHC. More than $60 \%$ of $B^{\Delta I} / B^{-}$mice developed overt hydrocephalus and showed more severe defects in neural cell migration and adhesion than did $B^{\Delta I} / B^{\Delta I}$ mice. These data on $B^{\Delta I} / B^{\Delta I}$ and $B^{\Delta I} / B^{-}$mice demonstrate a gene dosage effect of the amount of NMHC-B on the severity and time of onset of the defects in the heart and brain.

J. Clin. Invest. 105:663-671 (2000).

\section{Introduction}

Recent developments in molecular genetic techniques have made it possible to use mice as tools to better understand the genesis of human disease. This has resulted in the identification of many important genes that play major roles in the development of various diseases (1). For example, mutations in several contractile proteins such as the cardiac $\beta$-myosin heavy chain ( $\beta$-MHC; 2,3$)$, cardiac $\alpha$-actin $(4,5)$, and myosin light chains (6) are associated with cardiomyopathy. We have shown the essential role of nonmuscle MHC II-B (NMHC-B) in cardiac development (7). One difficulty in studying NMHC-B-ablated mice is that they die either during embryogenesis or on the day of birth (P0), making it impossible to analyze the postnatal progression of pathological processes. We addressed this problem by producing mice that express decreased levels of NMHC-B.

Nonmuscle myosins are class II myosins that are found in both muscle and nonmuscle cells. Previous work has suggested their involvement in basic cell functions such as cytokinesis (8), cell motility (9), formation of cell polarity (10), and changes in cell shape (11-13). They are composed of a pair of 200$\mathrm{kDa}$ heavy chains and 2 pairs of light chains $(20 \mathrm{kDa}$ and $17 \mathrm{kDa}$ ). Two different genes have been identified that encode the heavy chains: NMHC-A (MYH9)and NMHC-B (MYH10). Distinct cellular localizations and tissue distributions, and different enzymatic activities for NMHC-A and NMHC-B, suggest but do not prove that each protein may have specific functions (14-16). For example, although NMHC-A and NMHC-B are present in most cells, NMHC-B appears to be the only form present in murine cardiac myocytes (17). The pre-mRNA encoding NMHC-B, unlike that encoding NMHC-A, can undergo neural cell-specific alternative splicing, which results in the insertion of 10 amino acids near the ATP-binding region and 21 amino acids near the actin-binding region (18-20). The insert of 10 amino acids beginning after Pro ${ }^{211}$ is encoded by a neuralspecific exon referred to as N30 (see Figure 1), and is called the B1 insert. Expression of this insert is associated with neural differentiation, as is that of the B2 

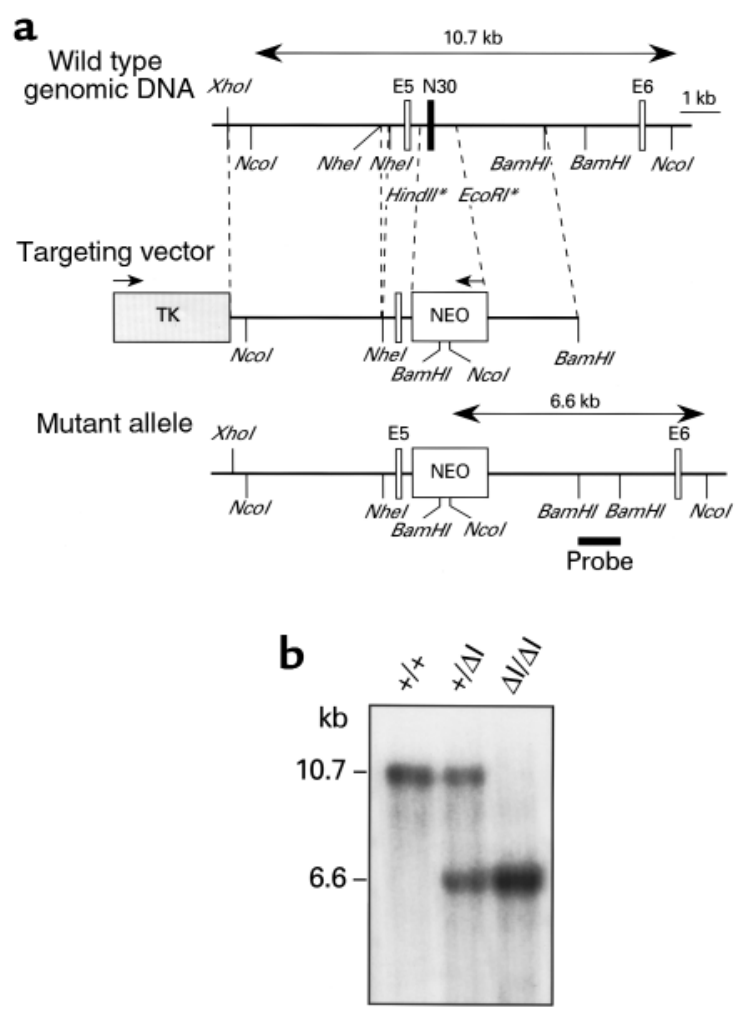

Figure 1

Generation and genomic analysis of $B^{\Delta I} / B^{\Delta I}$ mice. (a) Genomic map of the NMHC-B locus surrounding the N30 neural-specific alternative exon. E5 and E6 are constitutive exons encoding part of the ATP-binding region of NMHC-B. The HindII-EcoRI fragment, which includes the N30 exon, was replaced by the PGK-Neo gene. The PGK-Neo cassette (NEO) and the herpes simplex thymidine kinase gene (TK) are indicated by boxes. Arrows above the boxes indicate the direction of transcription. Some restriction sites for enzymes marked with an asterisk are not shown. Exons are not drawn to scale. (b) Southern blot analysis of genomic DNA isolated from mouse tails. Ncol was used to generate a 10.7-kb fragment indicating the wild-type allele and a $6.6-\mathrm{kb}$ fragment indicating the mutant allele. The probe used for Southern analysis is shown below the mutant allele in $\mathbf{a}$.

insert of 21 amino acids. However, the 2 inserts differ in that the B1 insert appears early in embryonic development, whereas the B2 insert appears only after birth (19). In this paper, we refer to NMHC-B that contains the $\mathrm{B} 1$ insert as the inserted form, and to that without the insert simply as NMHC-B.

We reported previously that ablation of NMHC-B in mice results in lethality during embryonic development or on P0. These mice showed major developmental defects in the heart and central nervous system. The cardiac defects included a membranous ventricular septal defect (VSD), dextroposition of the aorta, and myocyte hypertrophy. In addition, all the $B^{-} / B^{-}$mice developed hydrocephalus (7).

In this study, we report on cardiac and brain defects in 2 groups of mutant mice that show decreased levels of NMHC-B. One group of mice was generated by replacement of the N30 exon encoding the B1 insert with a cassette conferring neomycin resistance. In addition to ablating the neural-specific inserted form of mRNA, this replacement resulted in decreased amounts of NMHC-B mRNA and protein in all tissues. We have designated these mice $B^{\Delta I} / B^{\Delta I} ; \Delta \mathrm{I}$ refers to the deleted neural-specific exon. We then bred the $B^{\Delta I} / B^{\Delta I}$ mice with $B^{+} / B^{-}$mice to generate another group of mutant mice $\left(B^{\Delta I} / B^{-}\right)$with even less NMHC$B$. By comparing the cardiac and brain defects in $B^{\Delta I} / B^{\Delta I}$ and $B^{\Delta I} / B^{-}$mice to those of the previously described $B^{-} / B^{-}$mice, we establish a direct relationship between the decrease in NMHC-B and the severity of the defects. Moreover, the generation of $B^{\Delta I} / B^{\Delta I}$ and $B^{\Delta I} / B^{-}$mice allowed us to study the function of NMHC-B beyond embryonic development.

\section{Methods}

Generation of transgenic animals and Southern blot analysis. NMHC-B clones spanning exons 5 (constitutive), N30 (alternative), and 6 (constitutive) and flanking introns were isolated from a mouse (129Sv) genomic $\lambda$ FIXII library (Stratagene, La Jolla, California, USA) using human NMHC-B cDNA as a probe. As shown in Figure 1 , approximately $8 \mathrm{~kb}$ of a $\mathrm{XhoI}-\mathrm{Bam} \mathrm{HI}$ fragment was subcloned in pBluescript II SK+ (Stratagene) for use as a targeting vector. A $0.8-\mathrm{kb} H$ indII-EcoRI fragment, which consisted of the 30-bp N30 exon, and the flanking introns of approximately 250 bp upstream and 550 bp downstream, was replaced by a neomycin phosphotransferase (Neo) gene cassette (PGK-Neo) in the reverse orientation. A herpes simplex thymidine kinase gene cassette was also introduced at the $5^{\prime}$ end of the genomic fragment. Transcripts of Neo and thymidine kinase are driven by the mouse $P G K-1$ gene promoter and include the polyadenylation signal region of the PGK-1 gene. The targeting construct lacked a 200-bp NheI-NheI intron fragment located approximately 400 bp upstream of exon 5 . The linearized targeting vector was electroporated into J1 embryonic stem (ES) cells (a gift of R. Jaenisch, Whitehead Institute for Biomedical Research, Cambridge, Massachusetts, USA), and ES cell clones were selected by resistance to G418 and ganciclovir. Targeted ES cell clones were identified by Southern blot analysis after $\mathrm{NcoI}$ digestion, using a BamHI$B a m \mathrm{HI}$ fragment located outside the targeting vector as a probe (Figure 1). Recombinant ES cells were injected into blastocysts, resulting in male chimeras that were mated to C57BL/6J females to produce heterozygous offspring. Homozygous mice were generated by breeding $B^{+} / B^{\Delta I} \times B^{+} / B^{\Delta I}$ and $B^{\Delta I} / B^{\Delta I} \times B^{\Delta I} / B^{\Delta I}$ mice. $B^{\Delta I} / B^{\Delta I}$ mice were also bred with $B^{+} / B^{-}$mice to generate $B^{\Delta I} / B^{-}$ progeny. Genomic DNA from $B^{\Delta I} / B^{-}$mice was digested with BamHI, and the Southern blot was analyzed using a probe near exon 2 , as described previously, for identification of the $B^{-}$allele (7).

Immunoblot analysis. For comparison of the amounts of NMHC-B protein in $B^{+} / B^{+}, B^{+} / B^{\Delta I}, B^{\Delta I} / B^{\Delta I}, B^{+} / B^{-}$, and $B^{\Delta I} / B^{-}$mice, samples for immunoblot analysis were prepared using an extraction buffer containing $0.5 \mathrm{M}$ $\mathrm{NaCl}, 25 \mathrm{mM}$ Tris- $\mathrm{HCl}(\mathrm{pH}$ 7.5), $50 \mathrm{mM}$ sodium 
pyrophosphate, $5 \mathrm{mM}$ EDTA, $5 \mathrm{mM}$ EGTA, $10 \mathrm{mM}$ ATP, $5 \mathrm{mM}$ DTT, $5 \mu \mathrm{g} / \mathrm{L}$ leupeptin, $0.1 \mathrm{mM}$ PMSF, 10 $\mathrm{mg} / \mathrm{L}$ aprotinin, $1 \mathrm{mM}$ benzamidine, and an additional mix of proteolytic inhibitors $(25 \mu \mathrm{L}$ in $50 \mathrm{~mL}$ of extraction buffer: $0.1 \mathrm{~g}$ pepstatin $\mathrm{A}, 0.2 \mathrm{~g}$ chymostatin, $0.1 \mathrm{~g}$ TLCK, and $0.1 \mathrm{~g}$ TPCK in $10 \mathrm{~mL}$ DMSO). After centrifugation at $16,000 \mathrm{~g}$ for 15 minutes, the supernatants were recovered and gel electrophoresis was performed in SDS $6 \%$ or $7.5 \%$ polyacrylamide gels from Novex (San Diego, California, USA). After transfer to Immobilon-P membranes (Millipore Corp., Bedford, Massachusetts, USA), polyclonal antibodies specific for the carboxyl terminus of NMHC-A and NMHC-B were used for detection (21) with the SuperSignal or UltraSignal system from Pierce Chemical Co. (Rockford, Illinois, USA). A polyclonal antibody specific for the carboxyl terminus of the cardiac $\beta$-MHC was a gift of Neal Epstein (National Heart, Lung, and Blood Institute). Monoclonal antibodies raised against actin (Roche Molecular Biochemicals, Indianapolis, Indiana, USA) and GAPDH (BIODESIGN International, Kennebunk, Maine, USA) were used to confirm equal sample loading. Immunoblots were quantitated using a Personal Densitometer from Molecular Dynamics (Sunnyvale, California, USA).

Histological analysis of heart. Hearts were fixed with 3.7\% paraformaldehyde in $0.1 \mathrm{M}$ phosphate buffer ( $\mathrm{pH}$ 7.4), and were examined with a dissecting microscope to evaluate their external morphology. The hearts were then cut in half vertically with a razor blade to obtain views of the 4 chambers (echocardiographic cut) and were examined again with a dissecting microscope. They were then embedded in paraffin, sectioned, and stained with hematoxylin and eosin (H\&E) or with periodic acid-methenamine silver. To evaluate the degree of cardiac hypertrophy, the transverse diameters of the ventricular myocytes were measured $(n=30)$ as described previously (7). The magnification of the microscope used for measurements was calibrated with a stage micrometer. The mean and SD of these measurements was calculated for individual animals and for each group of animals. Data were analyzed using the 2tailed Student's $t$ test; $P<0.05$ was considered significant. The presence of a VSD was evaluated by examining serial sections of the area of the ventricular septum and the semilunar valves.

Histological analysis of brain. To prepare brains for histological analysis, the thoracic cavity of mice 3-4 weeks old was opened under pentobarbital anesthesia (30 $\mathrm{mg} / \mathrm{kg}$, given intraperitoneally). The right atrium was cut, and cold PBS was slowly perfused through the left ventricle with a 25 -gauge needle while the heart was still beating, until the fluid coming out of the right atrium was clear. Then about $5 \mathrm{~mL}$ of Bouin's fixative (EM Science, Gibbstown, New Jersey, USA) was perfused through the left ventricle to assure that the brain was well fixed. The brains were then dissected out and immersed in Bouin's fixative overnight.

For preparation of the P0 brain sections, mice were sacrificed on the day of birth by decapitation. The skin and part of the skull was opened at the midline, exposing the brain. The whole head was then immersed in Bouin's fixative overnight, and the brain was dissected out. All of the brain samples were embedded in paraffin, sectioned, and then stained with H\&E.

Magnetic resonance microscopy. After the mice were euthanized with an overdose of pentobarbital, images were obtained at 4.7 teslas on a GE Omega CSI instrument with a custom-designed microimaging birdcage coil (22).

Blood pressure measurements. Arterial blood pressure was measured in Avertin-anesthetized mice with a catheter in the abdominal aorta using a Model 200 Digi-Med Blood Pressure Analyzer (Micro-Med Inc., Louisville, Kentucky, USA).

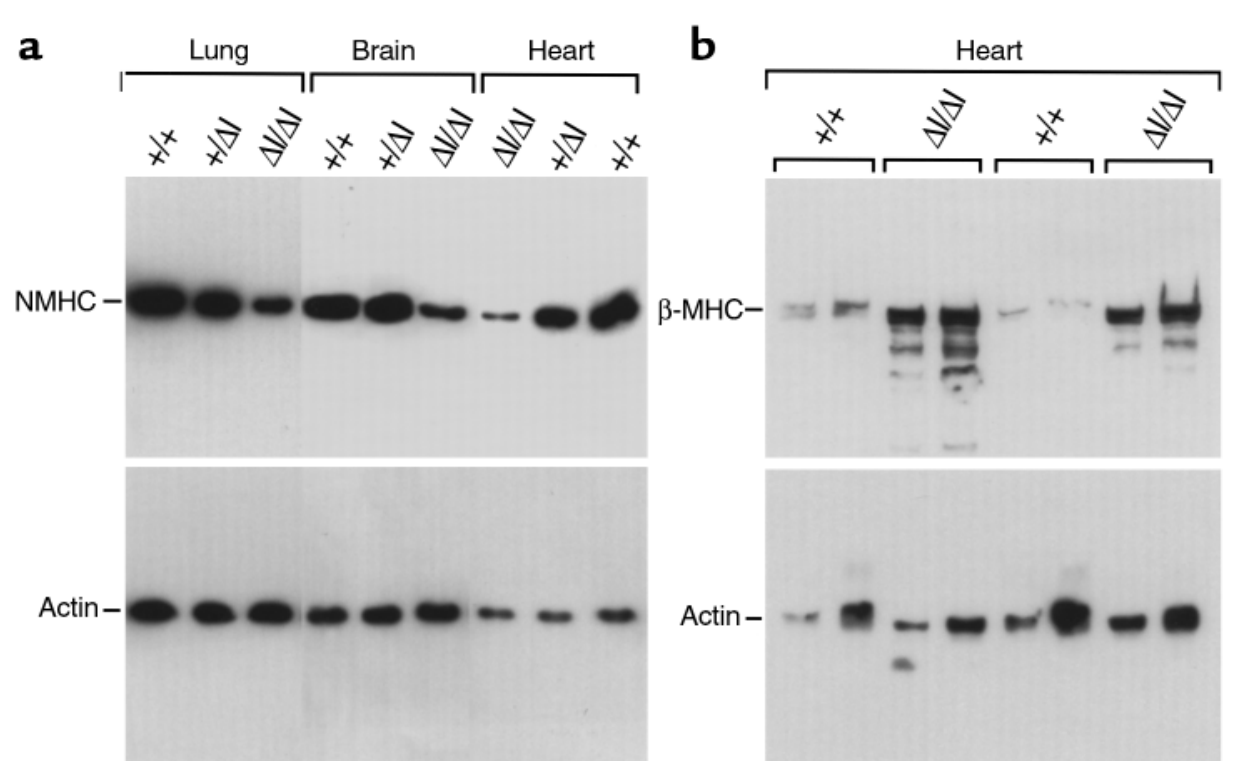

\section{Figure 2}

Immunoblot analysis of tissues from E14.5 and 11-month-old mice. (a) Comparison of NMHC-B in various tissues from $B^{+} / B^{+}, B^{+} / B^{\Delta I}$, and $B^{\Delta I} / B^{\Delta I}$ mice at E14.5. NMHC-B levels are decreased by $65 \pm 7 \%$ in the brain ( $n=$ 4) and by $88 \pm 4 \%$ in the heart $(n=5)$ of $B^{\Delta I} / B^{\Delta I}$ mice compared with $B^{+} / B^{+}$ littermates. The decrease in the lung sample shown is $64 \%$. (b) Reexpression of the fetal gene program showing a more than 5 -fold increase in the cardiac $\beta-\mathrm{MHC}$ in 2 different $B^{\Delta I} / B^{\Delta I}$ mice at 11 months of age. Two different loadings are shown for each sample. The immunoblots were overexposed to permit comparison with $B^{+} / B^{+}$samples. Quantitation was normalized using an antibody to actin (shown) and GAPDH (not shown) after $\mathrm{B}^{+} / \mathrm{B}^{+}$samples. 


\begin{abstract}
Figure 3
Myocyte size measurements and histological sections of $B^{+} / B^{+}$ and $B^{\Delta I} / B^{\Delta I}$ mouse hearts. (a) Plot of transverse diameters of cardiac myocytes vs. age of mice ( $n=3$ for each point; $P<0.05$ at 11 months of age). (b) Representative sections of a $B^{+} / B^{+}$ mouse heart (left) and a $B^{\Delta I} / B^{\Delta I}$ mouse heart (right) stained with periodic acid-methenamine silver, demonstrating cardiac hypertrophy at age 11 months. Bar, $10 \mu \mathrm{m}$
\end{abstract}
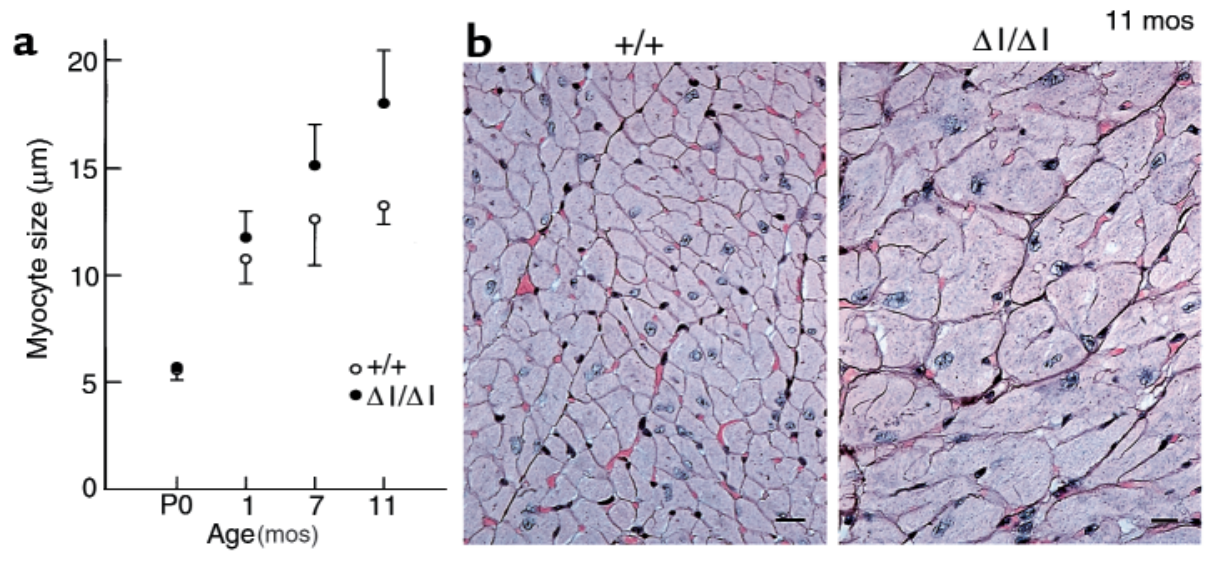

\section{Results}

Generation of B1 insert-deleted mice. Using the targeting strategy shown in Figure 1a, the N30 exon and $0.8 \mathrm{~kb}$ of the surrounding 2 introns were replaced with the PGK-Neo gene cassette by homologous recombination in mouse ES cells. Successfully targeted ES cell clones were identified by Southern blot analysis using the probe indicated in Figure 1a to distinguish an NcoI fragment of the mutant allele $(6.6 \mathrm{~kb})$ from that of the wild-type allele $(10.7 \mathrm{~kb})$. These clones were used to produce the chimeric males that gave rise to germline transmission of the mutant allele. Heterozygous $\left(B^{+} / B^{\Delta I}\right)$ mice were subsequently crossed to obtain homozygous $\left(B^{\Delta I} / B^{\Delta I}\right)$ progeny (Figure $1 \mathrm{~b}$ ). Deletion of the 30-nucleotide exon from NMHC-B mRNA was confirmed by RNA analysis of neural tissues.

Decreased NMHC-B protein in $B^{\Delta I} / B^{\Delta I}$ mice. Analysis of mRNA isolated from a variety of tissues from $B^{\Delta I} / B^{\Delta I}$ mice using a $3^{\prime}$ UTR probe specific for NMHC-B revealed a decrease in the NMHC-B message; no significant change was found in mRNA encoding NMHC-A (data not shown). This suggested that the replacement of the N30 exon by the PGK-Neo cassette resulted in a decrease in the steady-state level of NMHC-B, in addition to absence of the neural-specific exon. The decrease in NMHC-B mRNA resulted in a marked decrease in NMHC-B protein in $B^{\Delta I} / B^{\Delta I}$ tissues compared with $B^{+} / B^{+}$and $B^{+} / B^{\Delta I}$ tissues, in both embryonic (Figure 2a) and adult mice. There was no change in the amount of NMHC-A (data not shown).

Cardiac defects in $B^{\Delta I} / B^{\Delta I}$ mice. Previous work has shown that $B^{-} / B^{-}$mice develop consistent cardiac abnormalities, including enlarged cardiac myocytes, as early as E12.5 (7). Therefore, it was of particular interest to examine cardiac development in the $B^{\Delta I} / B^{\Delta I}$ mice, which demonstrated a marked decrease in NMHC-B. As shown in Figure 3a, $B^{\Delta I} / B^{\Delta I}$ mice were born with normalsized cardiac myocytes, but developed significant myocyte hypertrophy over a period of 11 months. The ratio of heart weight to body weight for $B^{\Delta I} / B^{\Delta I}$ mice was $0.006 \pm 0.0015(n=5)$, compared with $0.004 \pm 0.0006(n$ $=5$ ) for $B^{+} / B^{+}$mice (all mice were between 9 and 11 months of age). Figure $3 b$ shows representative sections from 11-month-old $B^{+} / B^{+}$(left) and $B^{\Delta I} / B^{\Delta I}$ (right) hearts, stained with periodic acid-methenamine silver to outline the borders of the myocytes; hypertrophy is clearly present at this age. In contrast to the $B^{-} / B^{-}$mice, none of the $18 B^{\Delta I} / B^{\Delta I}$ mice analyzed showed evidence of a VSD or any other cardiac malformation. Moreover, there was no evidence of an increase in blood pressure in $B^{\Delta I} / B^{\Delta I}$ mice compared with $B^{+} / B^{+}$mice $(n=6$ for each group; data not shown). However, cardiac myocyte hypertrophy was accompanied by reexpression of the fetal gene program, as shown by the increase in cardiac $\beta$-MHC at age 11 months in $B^{\Delta I} / B^{\Delta I}$ mice (Figure $2 \mathrm{~b}$ ).

Brain defects in $B^{\Delta I} / B^{\Delta I}$ mice. $B^{\Delta I} / B^{\Delta I}$ mice were born with no obvious external abnormalities of the head. Approximately $4 \%$ of these mice ( 8 of 216 ) showed progressive enlargement of the head at approximately $2-3$ weeks of age, and became moribund at 3-4 weeks of age. Histological analysis of the brains from 2 mice in this group at P23 and P29 demonstrated severe hydrocephalus with marked enlargement of the lateral and third ventricles, and massive destruction of the ventricular and subventricular structures (Figure $4 a$ ). Coronal sections of these $B^{\Delta I} / B^{\Delta I}$ mouse brains also showed stenosis of the cerebral aqueduct that could have impaired the circulation of the cerebral spinal fluid (CSF) and resulted in enlargement of the lateral and third ventricles, but not the fourth ventricle (data not shown). We also analyzed $2 B^{\Delta I} / B^{\Delta I}$ mice that showed no overt evidence of hydrocephalus at P23. Figure $4 \mathrm{~b}$ shows an abnormality in the cerebral aqueduct of 1 of these mice. The ependymal cells on the left side of the cerebral aqueduct are not present, and a bundle of nerve fibers is aberrantly protruding into the ventricle, but the cerebral aqueduct is not completely closed. The absence of the ependymal cells could be due to a primary defect in the neuroepithelial cells lining the ventricular wall.

Of particular note is that $B^{\Delta I} / B^{\Delta I}$ mice also showed abnormalities in cell migration at the level of the fourth ventricle, in addition to the loss of ependymal cells (Figure 4, $c$ and d). A number of large neurons are present ectopically in the dorsal part of the brainstem. In normal brains of mice of this age, neurons this large should have migrated out of the ventricular zone to their target area. The presence of neurons of this size near the ventricular surface suggests a defect in cell migration. 
c $\Delta \mathrm{I} / \Delta \mathrm{I}$

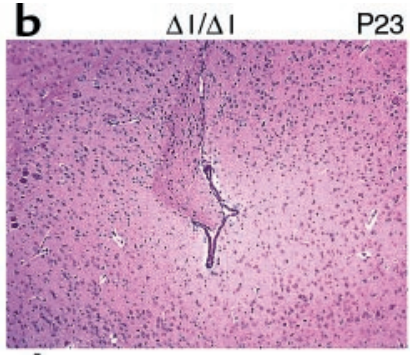

d

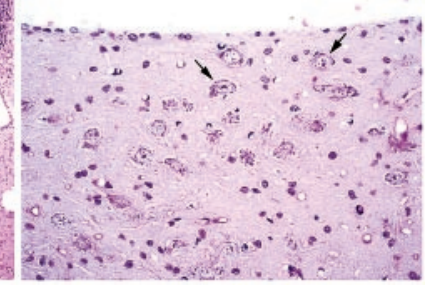

Figure 4

Brain defects in $B^{\Delta I} / B^{\Delta I}$ mice at P29 and P23. (a) Enlargement of lateral ventricles and destruction of the ventricular and subventricular structure in a P29 $B^{\Delta I} / B^{\Delta I}$ mouse. (b) Stenosis of the cerebral aqueduct with loss of the ependymal cells, and protrusion of a nerve fiber bundle in a $B^{\Delta I} / B^{\Delta I}$ mouse at P23. (c) Loss of the ependymal cells and misplaced large neurons at the level of the fourth ventricle in a $B^{\Delta I} / B^{\Delta I}$ mouse at P23. (d) Enlargement of the boxed area shown in c. A number of misplaced large neurons are present (arrows). Coronal sections; H\&E stain.

Generation of $B^{\Delta I} / B^{-}$mice. The appearance of cardiac myocyte hypertrophy in $B^{\Delta I} / B^{\Delta I}$ mice and the absence of the N30 exon in cardiac NMHC-B mRNA in $B^{+} / B^{+}$ mice (data not shown) strongly suggested that the cardiac phenotype observed in $B^{\Delta I} / B^{\Delta I}$ mice is due to the decrease in NMHC-B protein. To test this idea, we designed an experiment to further lower the amount of NMHC-B protein by crossing $B^{\Delta I} / B^{\Delta I}$ mice with $B^{+} / B^{-}$ mice to generate $B^{+} / B^{\Delta I}$ and $B^{\Delta I} / B^{-}$mice. Figure $5 \mathrm{a}$ com-

pares the amount of NMHC-B protein in newborn $B^{\Delta I} / B^{\Delta I}$ and $B^{\Delta I} / B^{-}$mice using immunoblot analysis of extracts from the lung, brain, and heart. The data show that all 3 tissues from $B^{\Delta I} / B^{-}$mice contain less NMHCB protein than do those of $B^{\Delta I} / B^{\Delta I}$ mice.

Cardiac defects in $B^{\Delta I} / B^{-}$mice. None of the $B^{\Delta I} / B^{\Delta I}$ mice showed a VSD or dextroposition of the aorta, both of which were seen in 7 of $8 B^{-} / B^{-}$mice (7). The refore, we were particularly interested in the cardiac phenotype of the $B^{\Delta I} / B^{-}$mice. Serial sections revealed a VSD at the level of the membranous septum in 5 of the $8 B^{\Delta I} / B^{-}$ mice examined (Figure 6a). Complete transposition of the great vessels accompanied the VSD in 1 of the $B^{\Delta I} / B^{-}$mice (data not shown). The other mice showed no abnormalities of the cardiac outflow tract.

Interestingly, newborn $B^{\Delta I} / B^{-}$mouse hearts showed evidence of myofibrillar disarray (Figure 6b) similar to that seen previously in $B^{-} / B^{-}$hearts, but no evidence of myocyte hypertrophy (7). However, in contrast to the 11 months required in $B^{\Delta I} / B^{\Delta I}$ mice, $B^{\Delta I} / B^{-}$mice developed myocyte hypertrophy and disarray by P24 (Figure 6c). At that time, the mean transverse diameter of the cardiac myocytes from $B^{\Delta I} / B^{-}$mice was $16.3 \pm 1.1 \mu \mathrm{m}$ $(n=5)$, compared with $11.8 \pm 0.9 \mu \mathrm{m}(n=10)$ for control mice $(P<0.001)$. As in the $B^{\Delta I} / B^{\Delta I}$ mice, the fetal gene program was also reinitiated in $B^{\Delta I} / B^{-}$mice, as shown by the marked increase in cardiac $\beta$-MHC by P23 (Figure 5b). Masson trichrome staining and electron microscopic analysis of $2 \mathrm{P} 24 B^{\Delta I} / B^{-}$mouse hearts showed no accumulation of collagen fibrils in the interstitium, confirming the lack of fibrosis in these hearts (data not shown).

Brain defects in $B^{\Delta I} / B^{-}$mice. $B^{\Delta I} / B^{-}$mice were born without overt evidence of hydrocephalus, but more than $60 \%$ of them developed progressive hydrocephalus with visible enlargement of the head and died at 3 to 4 weeks of age, compared with $4 \%$ of $B^{\Delta I} / B^{\Delta I}$ mice. The brains of $\mathbf{a}$
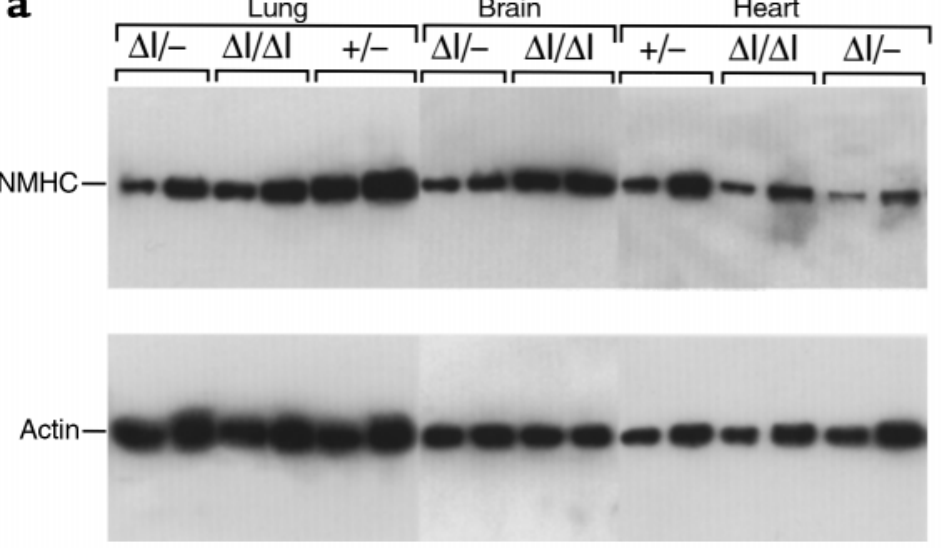

b

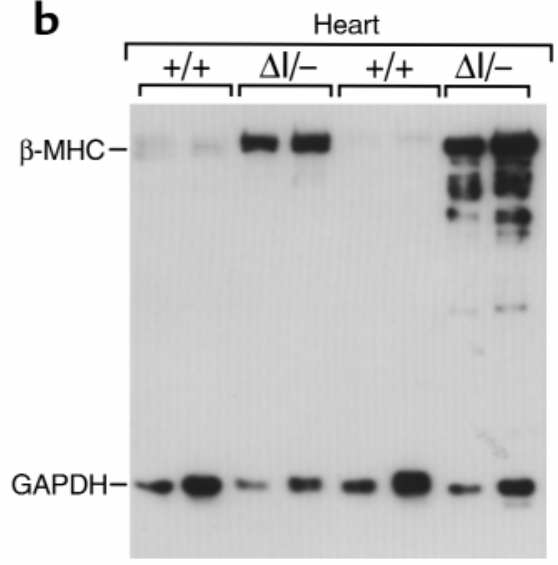

\section{Figure 5}

Immunoblot analysis of tissues from P0 and P23 mice. (a) Comparison of NMHC-B from $B^{\Delta I} / B^{-}, B^{\Delta I} / B^{\Delta I}$, and $B^{+} / B^{-}$mice at P0. Two different amounts were loaded for each sample. Quantitation was normalized using an antibody to actin. NMHC-B was decreased by $56 \pm 9 \%(n=4)$ and $55 \pm 5 \%(n=4)$ in brain and heart, respectively, compared with $B^{\Delta I} / B^{\Delta I}$ mice. The decrease in the lung sample shown is $25 \%$. (b) Reexpression of the fetal gene program in the hearts of $2 B^{\Delta I} / B^{-}$mice at $\mathrm{P} 23$ shows an approximately 40 -fold increase in upregulation of the cardiac $\beta$ $\mathrm{MHC}$ compared with $B^{+} / B^{+}$mice. Two different loadings are shown for each sample. Quantitation was normalized using an antibody to GAPDH. 
a
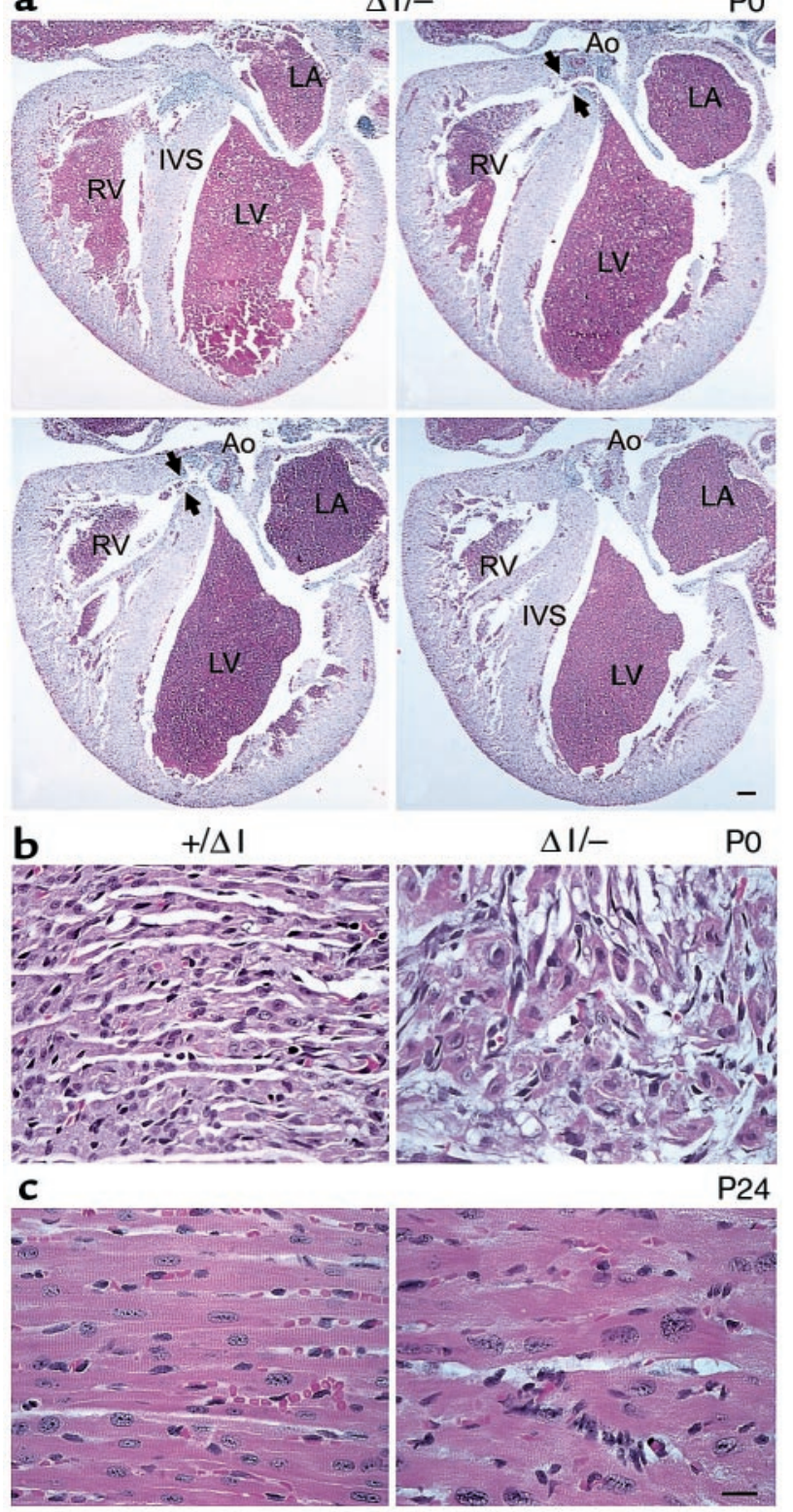

$\Delta \mathrm{I} /-$

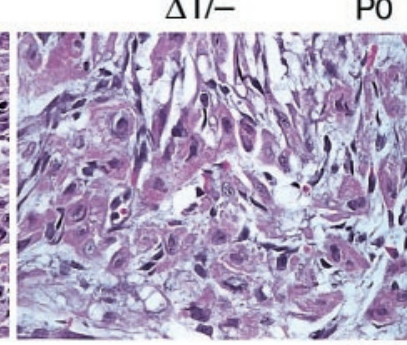

P24

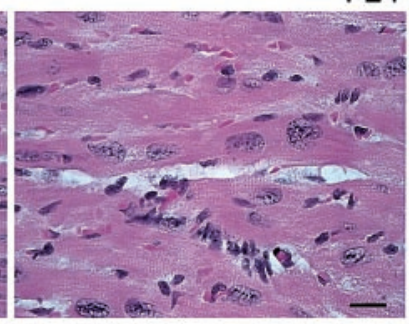

\section{Figure 6}

Histological sections of $B^{\Delta I} / B^{-}$and $B^{+} / B^{\Delta I}$ mouse hearts. (a) Four selected serial sections, anterior to posterior (clockwise from upper left), demonstrating the defect (arrowheads) in the membranous region of the ventricular septum in a $\mathrm{PO} B^{\Delta I} / B^{-}$mouse heart. Ao, aorta; IVS, interventricular septum; LA, left atrium; LV, left ventricle; RV, right ventricle. H\&E stain. Bar, $200 \mu \mathrm{m}$. (b) Normal cardiac histology in a $B^{+} / B^{\Delta I}$ mouse (left) and area of myocyte disarray and hyperchromatic nuclei in a $B^{\Delta I} / B^{-}$mouse (right). (c) Hypertrophied myocytes from a P24 $B^{\Delta I} / B^{-}$mouse (right) and myocytes from a $B^{+} / B^{\Delta I}$ littermate (left). H\&E stain. Bar, $20 \mu \mathrm{m}$.

$2 B^{\Delta I} / B^{-}$mice were analyzed at P24 and at 10 months of age using magnetic resonance microscopic imaging. Figure 7 shows images from a P24 $B^{\Delta I} / B^{-}$mouse (bottom panel) and a normal control (top panel). The brain of the $B^{\Delta I} / B^{-}$mouse shows massive expansion of the lateral ventricles and marked thinning of the cerebral cortex, but the cerebral aqueduct was not expanded. Coronal sections from all $4 B^{\Delta I} / B^{-}$mice analyzed at P0 showed mild to severe abnormalities in the brain. The most severe case is shown in Figure 8, and demonstrates typical hydrocephalus with enlargement of the lateral ventricles and massive destruction of the cerebral cortex. The ventricular and subventricular structures of the cerebral cortex were destroyed by hydrocephalus, edema, and vascular infarcts (Figure 8, a-c). The cerebral aqueduct, surrounded by a box in Figure $8 \mathrm{c}$ and magnified in Figure 8e, has an abnormal, asymmetric structure. In this case, stenosis of the cerebral aqueduct was probably the major cause of the hydrocephalus. The fourth ventricle, unlike the lateral and third ventricles, was not dilated (Figure $8 \mathrm{~d}$ ).

At the level of the fourth ventricle, $B^{\Delta I} / B^{-}$mice also showed evidence of a defect in neural cell migration, but of a more severe type and at an earlier time than in $B^{\Delta I} / B^{\Delta I}$ mice. As seen in Figure $8 \mathrm{~d}$ and at a higher magnification in Figure 8f, the ependymal cells (indicated by an arrowhead) were lost from the left side of the ventricular surface near the midline, and a mass of cells containing large neurons (indicated by arrows) protruded into the fourth ventricle. These large neurons appear to be from the same subset of cells that were abnormally present in the same region of the P23 $B^{\Delta I} / B^{\Delta I}$ mouse (Figure $4 d$ ), but their number appears to be increased. The mass of protruding cells caused a marked deformation of the ventricular wall, distorting the ventricle into a canal-like structure, as seen in Figure 8f, below the mass at the midline.

In summary, the results of this study demonstrate an inverse correlation between the severity of the abnormal phenotype and the amount of NMHC-B protein in the developing heart and brain. The abnormal phenotype is most severe and is present at the earliest timepoint in $B^{-} / B^{-}$mice. It is somewhat less severe in $B^{\Delta I} / B^{-}$ mice, and is least severe in $B^{\Delta I} / B^{\Delta I}$ mice.

\section{Discussion}

The original intent of these experiments was to generate mice that would lack the B1 inserted form of NMHC-B. Therefore, we replaced the $\mathrm{N} 30$ exon and $0.8 \mathrm{~kb}$ of the 2 flanking introns with the PGK-Neo cassette in a manner that potentially would not affect the expression of NMHC-B. However, analysis of $B^{\Delta I} / B^{\Delta I}$ mice showed a decrease in the mRNA encoding NMHC-B, as well as in the protein. This could have resulted from interference with the transcription of NMHC-B by the PGK-Neo cassette, as reported previously for other gene-targeted loci $(23,24)$, or from a decrease in RNA stability and processing. In addition, we cannot rule out the possibility that, in removing the flanking intron sequence to generate the targeting vector, we also removed an enhancerlike element. The marked decrease in NMHC-B protein concomitant with the decrease in mRNA is in contrast to the findings of others studying sarcomeric myosin, where protein levels were not affected by changes in transcript expression (25).

The $B^{\Delta I} / B^{\Delta I}$ mice were particularly useful, because all the $B^{-} / B^{-}$mice that we had previously generated died 
either during embryonic development or on $\mathrm{P} 0$, and because previous studies have shown that mice with decreased mRNA and protein expression (hypomorphs) can be quite informative $(23,24,26)$. These $B^{\Delta I} / B^{\Delta I}$ mice were able to reproduce and had a normal life span. Therefore, they were able to provide useful information about the effects of lowering NMHC-B below that of $B^{+} / B^{-}$mice, which appear to be normal. Crossing $B^{\Delta I} / B^{\Delta I}$ mice with $B^{+} / B^{-}$mice to produce $B^{\Delta I} / B^{-}$mice allowed direct testing of a gene dosage effect by further decreasing the amount of NMHC-B. These 3 groups of mice provide information on the function of NMHC-B during embryonic and postnatal development as well as during adult life. The major defects in both $B^{\Delta I} / B^{\Delta I}$ and $B^{\Delta I} / B^{-}$mice were confined to the heart and brain, similar to the defects in $B^{-} / B^{-}$mice. It is important to note that there was no evidence of increased blood pressure in $B^{\Delta I} / B^{\Delta I}$ mice compared with $B^{+} / B^{+}$mice. Moreover, inspection of the kidneys of $3 B^{\Delta I} / B^{\Delta I}$ and $2 B^{\Delta I} / B^{-}$mice that demonstrated cardiac myocyte hypertrophy showed no vascular changes of the type associated with hypertension (data not shown).

Cardiac defects. The $B^{-} / B^{-}$mice manifested 3 major defects: cardiac myocyte hypertrophy appearing as early as E12.5, a membranous VSD, and dextroposition of the aorta (7). $B^{\Delta I} / B^{\Delta I}$ mice were born with normal-sized cardiac myocytes and developed myocyte hypertrophy between 7 months and 11 months of age. Lowering NMHC-B by generating $B^{\Delta I} / B^{-}$mice had 3 apparent effects in these mice: it shortened the time required to generate myocyte hypertrophy from 11 months to 1 month, it resulted in a membranous VSD in 5 of 8 mice, and it led to the development of myofibrillar disarray, a finding that we noted previously in $B^{-} / B^{-}$mice (7). Only a single case (1 of 8) of an abnormality in the origin of the great vessels was found, showing that the VSD could develop in the absence of an abnormality in the outflow tract.

With respect to the findings of myofibrillar disarray, which was noted in both the $B^{-} / B^{-}$and $B^{\Delta I} / B^{-}$mouse hearts, recent work using immunofluorescence and confocal microscopy has shown that NMHC-B can be localized to the Z-lines and the intercalated discs (K. Takeda et al., manuscript submitted for publication). This suggests that NMHC-B plays a structural and possibly an active role in elements that are involved in the transmission of force and the maintenance of myocyte integrity during and after contraction. Absence $\left(B^{-} / B^{-}\right.$ mice) or a severe reduction $\left(B^{\Delta I} / B^{-}\right.$mice) of NMHC-B could disrupt normal myofibrillar structure and thus contribute to a compensatory cardiac hypertrophy. On the other hand, a smaller decrease in NMHC-B, as seen in the $B^{\Delta I} / B^{\Delta I}$ mice, might still allow hypertrophy to develop slowly by causing less impairment of the myocyte structure than that seen in $B^{\Delta I} / B^{-}$mice.

Work in other laboratories has shown that integrin signaling (27) and small GTPase proteins (28) play a role in the pathogenesis of cardiac myocyte hypertrophy. Because integrin signals are thought to be transmitted through cytoskeletal proteins, including nonmuscle actin and actin-binding proteins, it is possible that the decreased amounts of NMHC-B in the Z-lines and intercalated discs of the $B^{\Delta I} / B^{\Delta I}$ and $B^{\Delta I} / B^{-}$mice results in disruption of the normal integrin signaling system, and causes a compensatory increase in myocyte size. Unlike most vertebrate cells, cardiac myocytes contain only NMHC-B and not NMHC-A (17), and thus they are particularly vulnerable to decreased amounts of this protein. The delayed appearance of cardiac hypertrophy in $B^{\Delta I} / B^{\Delta I}$ mice is consistent with a milder defect, which is manifested only after prolonged cardiac contractile activity; it is similar in this respect to the defects seen after ablation of desmin (29).

Of particular note was the absence of a membranous VSD in $B^{\Delta I} / B^{\Delta I}$ mice and its presence in $B^{\Delta I} / B^{-}$and $B^{-} / B^{-}$ mice. This supports the idea that the cardiac myocyte hypertrophy seen in $B^{-} / B^{-}$and $B^{\Delta I} / B^{-}$mice is not dependent on the presence of a VSD. VSD is the most common congenital heart defect, and a membranous defect is the most frequent type. It is attributable to a failure in the closure of the secondary interventricular foramen (30). It is possible that a failure in migration of a subset of cells from the adjacent conotruncal septum and atrioventricular cushions may result in the septal defect, due to a decrease or complete absence of NMHC-B in these cells. The possibility that these cells are derived from neural crest cells cannot be ruled out, although it is

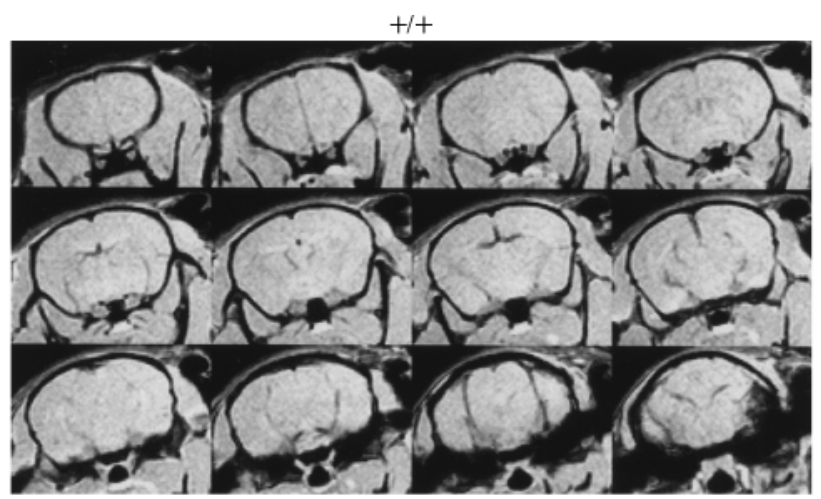

$\Delta \mathrm{l} /-$

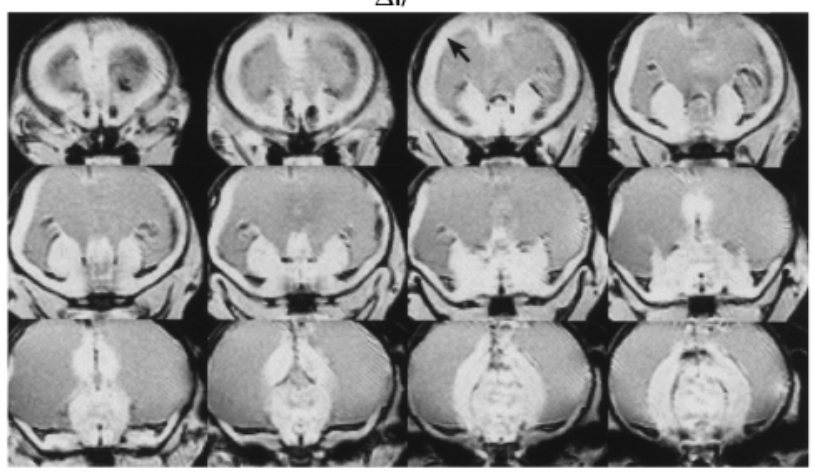

\section{Figure 7}

Magnetic resonance microscopy images of brain from a normal wildtype mouse (top) and a P24 $B^{\Delta I} / B^{-}$mouse (bottom). The images in the bottom panel show massive enlargement of the lateral ventricles and thinning of the cerebral cortex (arrow) due to hydrocephalus. 

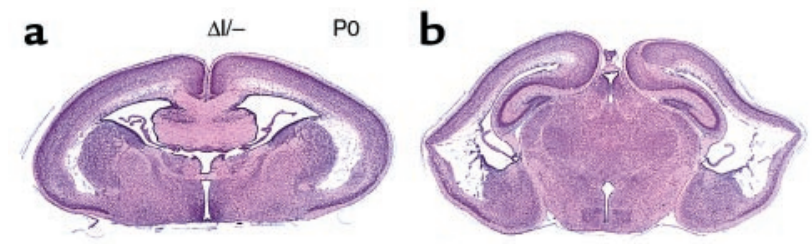

C
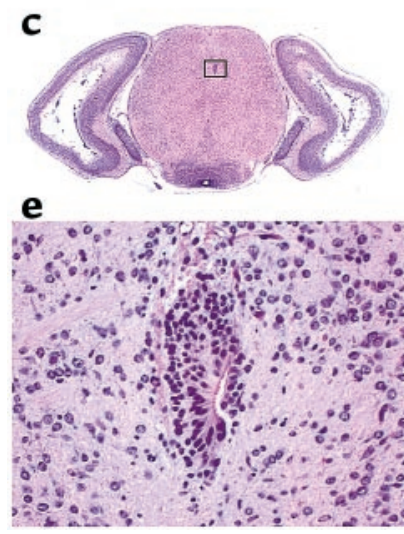

d
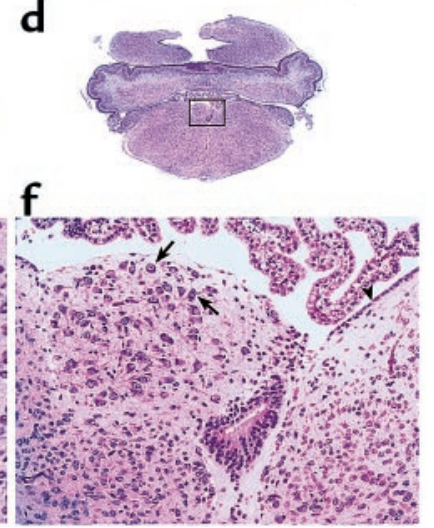

Figure 8

Brain defects in $B^{\Delta I} / B^{-}$mice at P0. (a-d) Low-magnification view of coronal sections from anterior to posterior. The lateral ventricles are expanded and the ventricular and subventricular structures of the cerebral cortex are destroyed (a-c), but the fourth ventricle is not expanded (d). The choroidal plexus looks normal (a, b, and d). (e) Enlargement of the boxed area shown in c showing stenosis and deformity of the cerebral aqueduct and the asymmetric development of the neuroepithelial cells. (f) Enlargement of the boxed area shown in $\mathbf{d}$, showing loss of ependymal cells and the abnormal protrusion of a mass of cells containing large neurons into the fourth ventricle. The misplaced large neurons (arrows) are present in abundance. The ependymal cell layer is indicated (arrowhead). H\&E stain.

unlikely because the integrity of the pulmonary artery and aorta is always maintained, even in $B^{-} / B^{-}$mice (31). Brain defects. The $B^{\Delta I} / B^{\Delta I}$ and $B^{\Delta I} / B^{-}$mice were very useful in studying the function of NMHC-B during the postnatal development of the nervous system, and also in analyzing the pathogenesis of the postnatal neurological defects caused by the decreased amounts of NMHC-B. These mutant mice complemented the $B^{-} / B^{-}$ mice, which were useful in studying the role of NMHCB during embryonic development of the brain (A.N. Tullio et al., manuscript submitted for publication). The present study demonstrates that the level of NMHC-B is well correlated with the onset and severity of the hydrocephalus that arises during postnatal development. Mice that express lower levels of NMHC$B$ develop a severe form of hydrocephalus at an earlier stage of postnatal life, and with a higher frequency. In the extreme case of $B^{-} / B^{-}$mice with no NMHC-B, hydrocephalus develops during embryonic development and the mice die between E14 and P0.

There are multiple mechanisms that could result in hydrocephalus. The brain defect most frequently seen in all 3 groups of NMHC-B mutant mice was the abnormal development of the cerebral aqueduct. In most cases with severe hydrocephalus, the cerebral aqueduct was almost completely closed due to defor- mity of the aqueduct and destruction of the ventricular surface. The impairment of the CSF circulation caused by blockage of the cerebral aqueduct appeared to result in the expansion of the lateral and third ventricles, but not the fourth ventricle. However, it is also noteworthy that multiple defects in the nervous system preceded full development of hydrocephalus. One of the defects directly relevant to hydrocephalus was the abnormal development of the neuroepithelial cells lining the ventricular wall. In NMHC-B mutant mice, the destruction of the ventricular surface due to the weak adherence of these neuroepithelial cells to each other appears to be the major cause of the deformity of the cerebral aqueduct. The anterior part of the cerebral aqueduct is very narrow; therefore, a small deformity in this region could lead to blockage of the CSF circulation. However, it is also possible that defects in CSF secretion and absorption play a role in the generation of hydrocephalus. These and other causes will need to be ruled out in future experiments.

We also present evidence of a defect in neural cell migration (32) at the level of the fourth ventricle. In NMHC-B mutant mice, a unique subset of large neurons was present near the ventricular surface in the dorsal region of the brainstem. In normal animals, neurons of this size are not present in this region after cell migration is completed. Therefore, it is likely that a defect in cell migration or another related defect is responsible for misplacement of these neurons. Again, there is an inverse correlation between the number of misplaced neurons and the level of NMHC-B. Mice with the lower levels of NMHC-B show a larger number of misplaced neurons at this site.

In summary, we have demonstrated that by lowering the amount of NMHC-B mRNA and protein, we produced mice that permit the study of the gradual development of defects that can serve as valuable models for human abnormalities. These include cardiac myocyte hypertrophy, a membranous VSD, and a congenital form of hydrocephalus. In both the heart and the brain, it appears that there are subsets of cells that require NMHC-B for normal migration and cell-cell interaction. Thus, these mice are helpful in understanding the role of myosin II-B in the development and normal function of the heart and brain, and should be useful in studying relevant, related human diseases.

\section{Acknowledgments}

The authors thank the members of the Laboratory of Molecular Cardiology, National Heart, Lung, and Blood Institute (NHLBI) for their help and useful discussions, Samuel Chacko (University of Pennsylvania) for genomic clones, Joe Frank and Su Xu (National Institutes of Health) for the MRI analysis, and Antoine Smith (NHLBI) for technical assistance. The gene-targeted mice were generated in the laboratory of Heiner Westphal (National Institute of Child Health and Human Development). Donald Gardner (Veterinary Resources Program) reviewed the pathology slides and Randy 
Clevinger (NHLBI) performed the blood pressure measurements. Catherine S. Magruder provided expert editorial assistance. Y. Hara was supported in part by Special Coordination Funds of the Science and Technology Agency for Strategic Promotion System for Brain Science and by Health Science Research Grants of the Ministry of Health and Welfare for Specific Diseases (Intractable Hydrocephalus) (1999-SD-17) in Japan.

1. Chien, K.R. 1996. Genes and physiology: molecular physiology in genetically engineered animals. J. Clin. Invest. 97:901-909.

2. Watkins, H., Seidman, J.G., and Seidman, C.E. 1995. Familial hypertrophic cardiomyopathy: a genetic model of cardiac hypertrophy. Hum. Mol. Genet. 4:1721-1727.

3. Rayment, I., Holden, H.M., Sellers, J., Fananapazir, L., and Epstein, N.D 1995. Structural interpretation of the mutations in the $\beta$-cardiac myosin that have been implicated in familial hypertrophic cardiomyopathy. Proc. Natl. Acad. Sci. USA. 92:3864-3868.

4. Olson, T.M., Michels, V.V., Thibodeau, S.N., Tai, Y.-S., and Keating, M.T 1998. Actin mutations in dilated cardiomyopathy, a heritable form of heart failure. Science. 280:750-752.

5. Mogensen, J., et al. 1999. $\alpha$-cardiac actin is a novel disease gene in familial hypertrophic cardiomyopathy. J. Clin. Invest. 103:R39-R43.

6. Vemuri, R., et al. 1999. The stretch-activation response may be critical to the proper functioning of the mammalian heart. Proc. Natl. Acad. Sci. USA. 96:1048-1053.

7. Tullio, A.N., et al. 1997. Nonmuscle myosin II-B is required for normal development of the mouse heart. Proc. Natl. Acad. Sci. USA. 94:12407-12412.

8. De Lozanne, A., and Spudich, J.A. 1987. Disruption of the Dictyostelium myosin heavy chain gene by homologous recombination. Science. 236:1086-1091.

9. Svitkina, T.M., Verkhovsky, A.B., McQuade, K.M., and Borisy, G.G. 1997. Analysis of the actin-myosin II system in fish epidermal keratocytes: mechanism of cell body translocation. J. Cell Biol. 139:397-415.

10. Guo, S., and Kemphues, K.J. 1996. A non-muscle myosin required for embryonic polarity in Caenorhabditis elegans. Nature. 382:455-458.

11. Edwards, K.A., and Kiehart, D.P. 1996. Drosophila nonmuscle myosin II has multiple essential roles in imaginal disc and egg chamber morphogenesis. Development. 122:1499-1511.

12. Sellers, J.R. 1998. Myosins. 2nd edition. Oxford University Press. Oxford, United Kingdom.

13. Bresnick, A.R. 1999. Molecular mechanisms of nonmuscle myosin-II regulation. Curr. Opin. Cell Biol. 11:26-33.

14. Murakami, N., Mehta, P., and Elzinga, M. 1991. Studies on the distribution of cellular myosin with antibodies to isoform-specific synthetic peptides. FEBS Lett. 278:23-25.

15. Kelley, C.A., et al. 1996. Xenopus nonmuscle myosin heavy chain iso- forms have different subcellular localizations and enzymatic activities. J. Cell Biol. 134:675-687.

16. Kolega, J. 1998. Cytoplasmic dynamics of myosin IIA and IIB: spatial "sorting" of isoforms in locomoting cells. J. Cell Sci. 111:2085-2095.

17. Murakami, N., Trenkner, E., and Elzinga, M. 1993. Changes in expression of nonmuscle myosin heavy chain isoforms during muscle and nonmuscle tissue development. Dev. Biol. 157:19-27.

18. Takahashi, M., Kawamoto, S., and Adelstein, R.S. 1992. Evidence for inserted sequences in the head region of nonmuscle myosin specific to the nervous system. J. Biol. Chem. 267:17864-17871.

19. Itoh, K., and Adelstein, R.S. 1995. Neuronal cell expression of inserted isoforms of vertebrate nonmuscle myosin heavy chain II-B. J. Biol. Chem. 270:14533-14540.

20. Kawamoto, S. 1996. Neuron-specific alternative splicing of nonmuscle myosin II heavy chain-B pre-mRNA requires a cis-acting intron sequence. J. Biol. Chem. 271:17613-17616.

21. Phillips, C.L., Yamakawa, K., and Adelstein, R.S. 1995. Cloning of the cDNA encoding human nonmuscle myosin heavy chain-B and analysis of human tissues with isoform-specific antibodies. J. Muscle Res. Cell Motil. 16:379-389.

22. Xu, S., et al. 1998. In vivo three-dimensional MR microscopy of mice with chronic relapsing experimental autoimmune encephalomyelitis after treatment with insulin-like growth factor-I. AJNR Am. J. Neuroradiol. 19:653-658.

23. Fiering, S., et al. 1995. Targeted deletion of $5^{\prime}$ HS 2 of the murine $\beta$-globin LCR reveals that it is not essential for proper regulation of the $\beta$-globin locus. Genes Dev. 9:2203-2213.

24. Meyers, E.N., Lewandoski, M., and Martin, G.R. 1998. An Fgf8 mutant allelic series generated by Cre- and Flp-mediated recombination. Nat. Genet. 18:136-141.

25. Fewell, J.G., et al. 1999. Functional significance of cardiac essential light chain isoform switching in transgenic mice. J. Clin. Invest. 101:2630-2639.

26. Moens, C.B., Auerbach, A.B., Conlon, R.A., Joyner, A.L., and Rossant, J. 1992. A targeted mutation reveals a role for $\mathrm{N}-m y c$ in branching morphogenesis in the embryonic mouse lung. Genes Dev. 6:691-704.

27. Ross, R.S., et al. 1998. $\beta_{1}$ integrins participate in the hypertrophic response of rat ventricular myocytes. Circ. Res. 82:1160-1172.

28. Finkel, T. 1999. Myocyte hypertrophy: the long and winding RhoA'd. J. Clin. Invest. 103:1619-1620.

29. Li, Z., et al. 1997. Desmin is essential for the tensile strength and integrity of myofibrils but not for myogenic commitment, differentiation, and fusion of skeletal muscle. J. Cell Biol. 139:129-144.

30. Graham, T.P., Jr., and Gutgesell, H.P. 1995. Ventricular septal defects. In Heart disease in infants, children, and adolescents including the fetus and young adult. G.C. Emmanouilides, T.A. Riemenschneider, H.D. Allen, and H.P. Gutgesell, editors. Williams and Wilkins. Baltimore, MD. 724-746.

31. Kirby, M.L. 1999. Contribution of neural crest to heart and vessel morphology. In Heart development. R.P. Harvey and N. Rosenthal, editors. Academic Press. San Diego, CA. 179-193.

32. Hatten, M.E. 1999. Central nervous system neuronal migration. Annu. Rev. Neurosci. 22:511-539. 